\title{
Running-Wheel Avoidance Behavior in the Wistar/Kyoto Spontaneously Hypertensive Rat
}

\author{
ROBERT J. CAMPBELL ${ }^{1}$ AND LEO V. DI CARA \\ Mental Health Research Institute, University of Michigan, Ann Arbor, MI 48109
}

(Received 7 June 1976)

\begin{abstract}
CAMPBELL, R. J. AND L. V. DI CARA. Running wheel avoidance behavior in the Wistar/Kyoto spontaneously hypertensive rat. PHYSIOL. BEHAV. 19(4) 473-480, 1977. - Conditioned avoidance acquisition was studied in the Okamoto-Aoki spontaneously hypertensive rat (SHR) and in the Wistar/Kyoto (WKYN) strain from which SHRs were derived. Results have demonstrated that those rats within the WKYN foundation stock exhibiting higher systolic (tail) blood pressure (BP) also exhibit greater motor activity and superior levels of discriminated active avoidance learning in a running wheel. SHRs, and WKYNs with high BPs, acquired the CAR rapidly during the first of four sessions, taking very few pulsed shocks, whereas those WKYNs with the lowest (i.e., normotensive) BPs acquired the task more slowly, if at all. It is hypothesized that the increased pituitary-adrenocortical and sympatho-adrenomedullary activity previously reported for SHRs is genetically determined as an anticipatory hyperreactivity in hypertensive rats of the WKYN stock, is responsible at least in part, for the high level of running-wheel motor activity and CAR performance, and is a major contributing factor in the development of high blood pressure in these animals.
\end{abstract}

Active avoidance Blood pressure Pituitary-adrenocortical axis Running wheel activity Spontaneously hypertensive rat Sympathetic nervous system

IN SUMMARIZING the findings of ten years of research concerning the pathophysiological mechanism(s) involved in the development and/or maintenance of hypertension in the spontaneously hypertensive rat (SHR), it can be stated that a functional hyperreactivity of sympathetic nervous and endocrine systems, presumably due to a genetic disturbance, alters the peripheral vasculature functionally, as well as structurally, bringing about an increase in peripheral resistance and, consequently, higher systolic and diastolic blood pressures. Apparently, centrally controlled neuro-hormonal pressor influences, activated when animals are exposed to stressful or potentially stressful (anticipatory) stimuli, integrated via limbic and hypothalamicohypophyseal defense areas, and expressed peripherally, at least in part, through the sympathetic (adrenergicnoradrenergic) branch of the autonomic nervous system (SNS, including the adrenal medulla), are involved in the pathogenesis of hypertension $[5,12,13,22-24,28]$. The effects of hyperreactivity of the endocrine system thought to exist in SHR [23] may parallel the influence of this sympathetic over-activity. It has been reported that $\mathrm{SHR}$, as compared to Wistar/Kyoto (WKYN) controls, exhibit signs of functional hyperreactivity of the pituitary-adrenocortical axis, coinciding with decreased retention of the peptidergic hormones ACTH and vasopressin in the adenohypophysis and neurohypophysis, respectively [34].

In general, very little is known about the behavior of the SHR/WKYN strain. More specifically, attempts to obtain behavioral activity measures corresponding to (susceptibility to) hypertension and general overreactivity of sympathetic and endocrine function in SHR have yielded results which, because of discrepancies dependent on what control comparisons were made, allow less than unequivocal conclusions regarding any correlation between the two. These discrepancies in previous studies are likely due to strain differences inappropriately attributed to differential blood pressures. This important but too often neglected point, that the inbred ancestral WKYN normotensive rat is the only appropriate control for the SHR derived therefrom, has been made before $[9,10]$. Nevertheless, taking this into account, recent evidence suggests that SHRs display a higher behavioral level of locomotor activity as compared to normotensive control rats ([26,31], L. G. M. Wilson, private communication). For example, judging from most reports, SHRs are more active both in an open field and running-wheel activity test situation and thus appear to be less fearful of novel stimuli.

The fact that SHRs and WKYNs are genetically selected from the same foundation stock, for blood pressure (BP) and presumably sympathetic and peptidergic-hormonal activity extremes, is of major importance in any study comparing early behavioral correlates of these extremes. In certain investigations, where the BP parameter has been manipulated experimentally (e.g., stress, nutritional and/or surgical intervention), it is difficult to separate effects of the procedure used to change BP from the effects of BP per

\footnotetext{
' Present address: Department of Physiology, University of Southern California School of Medicine, Los Angeles, CA 90033.
} 
se. Thus, appropriately, the use of the SHR/WKYN-control set genetically selected for these extremes eliminates the confounding effect on behavior that experimental manipulation might have. For the same reason, study of the SHR/WKYN set is more meaningful at prehypertensive or early hypertensive than at later malignant stages. This reduces the likelihood that measured behavioral effects, defining a particular behavioral profile, are resulting from, rather than genetically coinciding with, or possibly even causing, hypertension per se.

According to the mediational hypothesis for avoidance learning $[21,33]$, initially an unconditioned noxious stimulus (US) elicits conditioned autonomic and other visceral responses with concomitant central alerting which later occur upon presentation of the conditioning (warning) stimulus (CS) and that the CAR, via this behavioral activation, alleviates these conditioned visceral responses, thereby providing tangible reinforcement. If a high level of sympathetic autonomic and peptidergic-hormonal reactivity can be equated with greater visceral responsivity and central alerting, the implications for such a hypothesis for avoidance learning are clear: animals exhibiting such a profile, coinciding with heightened motor activity, should presumably have less difficulty in, and/or reach a greater level of, acquisition and retention of the CAR. Conversely, animals derived from the same ancestral foundation stock, but exhibiting lower sympathetic and peptidergic-hormonal activity should exhibit poor active avoidance performance.

As a test of this hypothesis, conditioned running-wheel avoidance was studied in the WKYN-derived spontaneously hypertensive rat and its ancestral control, the WKYN normotensive rat. In addition, since so little is known about the behavior of rats within the Wistar-Kyoto foundation stock, this study serves to widen the data base for behavioral characterization of these animals.

\section{METHOD}

\section{Animals}

The animals were fourteen naive male Wistar-Kyoto (WKYN) rats and eleven age/weight-matched naive male spontaneously hypertensive rats (SHR) derived originally from the Wistar-K yoto strain by Okamoto and Aoki [23]. All animals were supplied by Bio-Lab Corporation (St. Paul, $\mathrm{MN})$ and housed individually in stainless steel cages ( $25 x$ $18 \times 17.5 \mathrm{~cm})$ at a room temperature of $23^{\circ} \mathrm{C}$ under a $12 \mathrm{hr}$ light-dark cycle for at least one month before testing at approximately 6-7 months of age (about 340-350 g) (Table 1). Food and water were available ad lib prior to and throughout the experiment.

\section{TABLE}

AGE, BODY WEIGHT AND TAIL BLOOD PRESSURE OF WISTAR/KYOTO-DERIVED RATS

\begin{tabular}{lccc}
\hline Group & $\begin{array}{c}\text { Age } \\
\text { Months }\end{array}$ & $\begin{array}{c}\text { Body Weight } \\
\text { Grams }\end{array}$ & $\begin{array}{c}\text { Tail Blood Pressure } \\
\mathrm{mm} \mathrm{Hg}\end{array}$ \\
\hline $\begin{array}{l}\text { SHR } \\
(\mathrm{n}=11)\end{array}$ & $6.2 \pm 0.4$ & $339 \pm 8.2$ & $200 \pm 3.8^{*}$ \\
$\begin{array}{l}\text { WKYN } \\
(\mathrm{n}=14)\end{array}$ & $6.5 \pm 0.2$ & $352 \pm 8.3$ & $144 \pm 6.9$ \\
\hline
\end{tabular}

All values expressed as mean \pm standard error of the mean.

*Significantly differs from WKYN $(p<0.001)$
Although classified as normotensive, the WKYN tran from Bio-Lab covered a wide range of BPs and some of these WKYNs had BPs ranging up to the lower values recorded from SHRs. The mean systolic BP values for the SHR and WKYN experimental groups are presented in Table 1. Initial statistical analysis (Student's $t$ ) of these data revealed significantly higher $(p<0.001)$ systolic BP in the SHRs $(200 \pm 3.8 \mathrm{~mm} \mathrm{Hg}, \mathrm{n}=11)$ as compared to WKYNs $(144 \pm 6.9 \mathrm{~mm} \mathrm{Hg}, \mathrm{n}=14)$, with only one rat of the WKYN strain having a $B P$ within the SHR range $(187.231 \mathrm{~mm}$ $\mathrm{Hg}$.

\section{Apparatus and Procedure}

The running-wheel, adapted from a lightweight bicycle wheel (axle spoked to rim), was $53 \mathrm{~cm}$ in diameter, with an external running surface consisting of aluminum bars ( $5 \mathrm{~mm}$ diameter, $14 \mathrm{~cm}$ long) attached rigidly to one side of the rim, spaced $18 \mathrm{~mm}$ apart. The rat, which ran on the outer perimeter of the wheel, was restrained within an open-top Plexiglas cubicle $(27 \times 13 \times 28 \mathrm{~cm}, 5 \mathrm{~mm}$ thick walls) (Fig. 1) by anchoring the adhesive-taped base of its tail softly, but securely through the rear wall of the restrainer. This arrangement also prevented the rat from reaching the tail-shock electrode fastened outside of the restrainer. Running-grid shock delivery to the rats' feet was not used because animals often alter shock delivery in avoidance-escape situations by means of postural changes [37]. The entire running-wheel apparatus was enclosed in a thick-walled, sound-attenuating chamber. The US was intermittent $\mathrm{AC}$ tail shock delivered through a solid state shocker (Lehigh Valley Electronics) with a current intensity of approximately $1.0 \mathrm{~mA}$ as measured with the rat in the circuit. The CS, delivered through a Mallory Sonalert $2.9 \mathrm{kHz}$ buzzer mounted parallel to the axis of the wheel toward the rear end of the chamber, was an $84 \mathrm{~dB}$ tone, as measured from within the Plexiglas wheel housing. A house light and a ventilating fan generating a background noise level of approximately $75 \mathrm{~dB}$ operated throughout adaptation and avoidance sessions. A silent photoelectric cell arrangement, through which movement of the runninggrid bars yielded an accurate measure of wheel revolutions ( 88 Bars $=1$ full wheel revolution), was wired to activate solid-state logic and counters outside the chamber. The presentation of stimuli and recording of responses were automated by BRS-Foringer programming equipment.

\section{Blood Pressure Screening}

At least 3 days prior to the first adaptation period, systolic blood pressure (BP) was measured in each rat using the same procedure and apparatus described in detail by Pfeffer et al. [27]. Briefly, indirect systolic pressures were recorded in prewarmed, pentobarbital-anesthetized (50 $\mathrm{mg} / \mathrm{kg}$ IP) rats by occluding, with an inflatable tail cuff, caudal artery blood flow and detecting, by means of a ventrally applied pulse transducer (Buffington Clinical Devices, Cleveland, $\mathrm{OH}$ ), the return of the pulse distal to the cuff upon deflation. Arterial pulse displacements were recorded on a Grass model 7B polygraph calibrated regularly with a Baum mercury manometer. A pproximately full scale displacements were obtained by maintaining ambient temperatures at $39 \pm 3^{\circ} \mathrm{C}$ by means of an infra red lamp during BP measurements. For each rat, a minimum of 2 sequences of 5-6 measurements were taken on each of 2-4 days. Reliability of the procedure was 


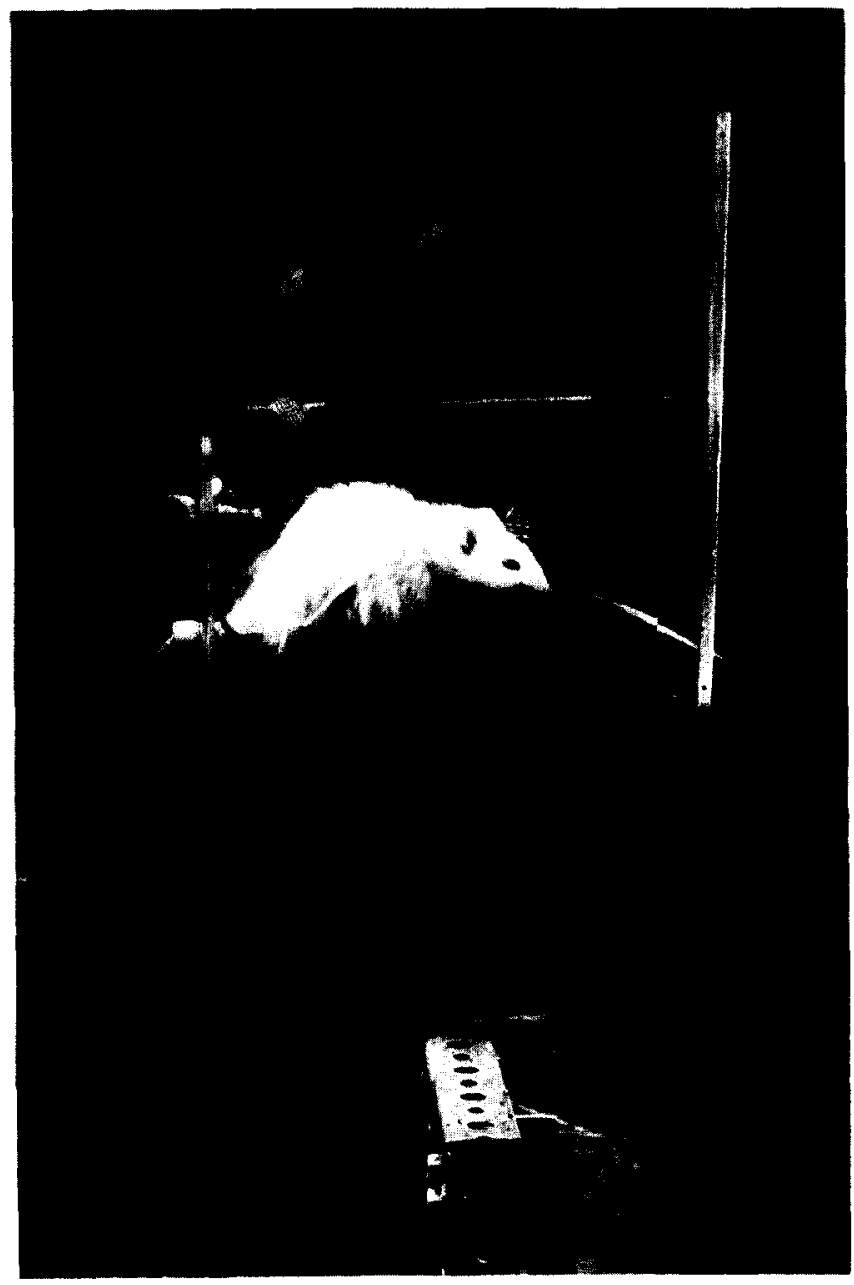

FIG. 1. Running-wheel apparatus for study of discriminated active avoidance in spontaneously hypertensive and normotensive rats. The rat is restrained in its running area on the outer perimeter of the wheel by securing the base of its tail through the rear wall of the Plexiglas housing, outside of which is fastened the tail-shock electrode.

previously determined by simultaneous direct (carotid artery reference) and indirect measurements under pentobarbital anesthesia. Bunag has shown that the carotid artery is the best reference for systolic pressure in the rat's tail [4]. The mean systolic pressures for 63 simultaneously recorded measurements ( \pm standard error of the mean) in 7 pentobarbital-anesthetized rats were $142.7 \pm 1.9$ for the direct carotid artery method $(\mathrm{X})$ and $143.4 \pm 1.8$ for the indirect tail-cuff method (Y). The correlation between these arterial pressure data pairs, over the range of $114-176 \mathrm{~mm} \mathrm{Hg}$, was quite significant $(\mathrm{r}=.933)$, with a regression coefficient near unity (.898) (Fig. 2).

Measurement of BP by this tail-cuff method in unrestrained, pentobarbital-anesthetized rats was chosen for this particular study, over measurement in restrained, unanesthetized animals for two reasons. First, depth of pentobarbital anesthesia was, in our hands, more easily controlled for than were the physiological and behavioral sequelae of physical restraint and the variable resistance/ reaction to that restraint in naive animals. Second, it appeared to us from earlier pilot studies that in the interest of obtaining clear and easily delimited recordings, too large and variable an amount of recording noise (e.g., movement artefact) resulting from the highly sensitive transducer precluded our use of the restrained, unanesthetized preparation. A representative recording is shown in Fig. 3. Numerous other reports confirm the reliability of such tail-cuff techniques for determining systolic blood pressure in anesthetized rats (seven references given in [27]).

\section{Avoidance Conditioning}

Rats were adapted to the enclosed running-wheel apparatus for two $30 \mathrm{~min}$ periods before the first CAR session. Each rat was run for one CAR session per day for 4 consecutive days. An avoidance session consisted of 60 trials and lasted approximately 1-2 hr. A standard signaled-avoidance procedure was employed: Each trial began with presentation of the CS for $5.0 \mathrm{sec}$, followed by onset of the US, a pulsed tail shock $(0.5 \mathrm{sec} /$ pulse $)$ every $2.0 \mathrm{sec}$, for $52 \mathrm{sec}$. Tone presentation was continued during the period of pulsed shock delivery. If the rat emitted a criterion wheel-running response (1/2 turn of the wheel for Days 1 and 2, 1 full turn for Days 3 and 4) during the initial tone presentation, the tone terminated without shock, i.e., a successful avoidance response ( $R a)$. If the rat failed to emit an $\mathrm{Ra}$ during the CS-US interval, pulsed shock was presented and continued until a successful wheel-running response (i.e., an escape response, $\mathrm{Re}$ ) was emitted, or until $52 \mathrm{sec}$ had elapsed, whichever occurred first, and at which time tone and shock coterminted. A variable intertrial interval (ITI) averaging $1 \mathrm{~min}$ (range $27 \mathrm{sec}$ to $90 \mathrm{sec}$ ) was given randomly between the end of one trial and the onset of the next trial.

\section{Data Analysis}

Kruskal-Wallis one-way analyses of variance were employed to test differences in avoidance responding, pulsed shock delivery and running activity during avoidance sessions. Following an overall significant effect, subsequent comparisons were made with Mann-Whitney U tests. Students' $t$-test was used where a parametric statistic was applicable (e.g., blood pressure, adaptation running score). Experimental design is outlined in Table 2, which also indicates sample size.

\section{RESULTS}

\section{Blood Pressure}

The initial BP data for the SHR and WKYN rats was presented under Animals and shown in Table 1 as two experimental groups based on the supplier's description, and by definition, on the genetic distinction between them. Nevertheless, subsequent behavioral data (see below) dictated that the WKYN group be subdivided for data analysis purposes into two separate subgroups, WKYN-1 (n $=6)$ and WKYN-2 $(n=8)$. BP values for these two groups, $123 \pm 4.9$ and $161 \pm 7.4$, respectively, were significantly less than that for SHRs $(p<0.001)$, and significantly differed from each other $(p<0.002)$ (Table 2$)$.

\section{Adaptation}

Table 2 shows each group's wheel-running activity recorded in two separate $30 \mathrm{~min}$ adaptation periods prior to 


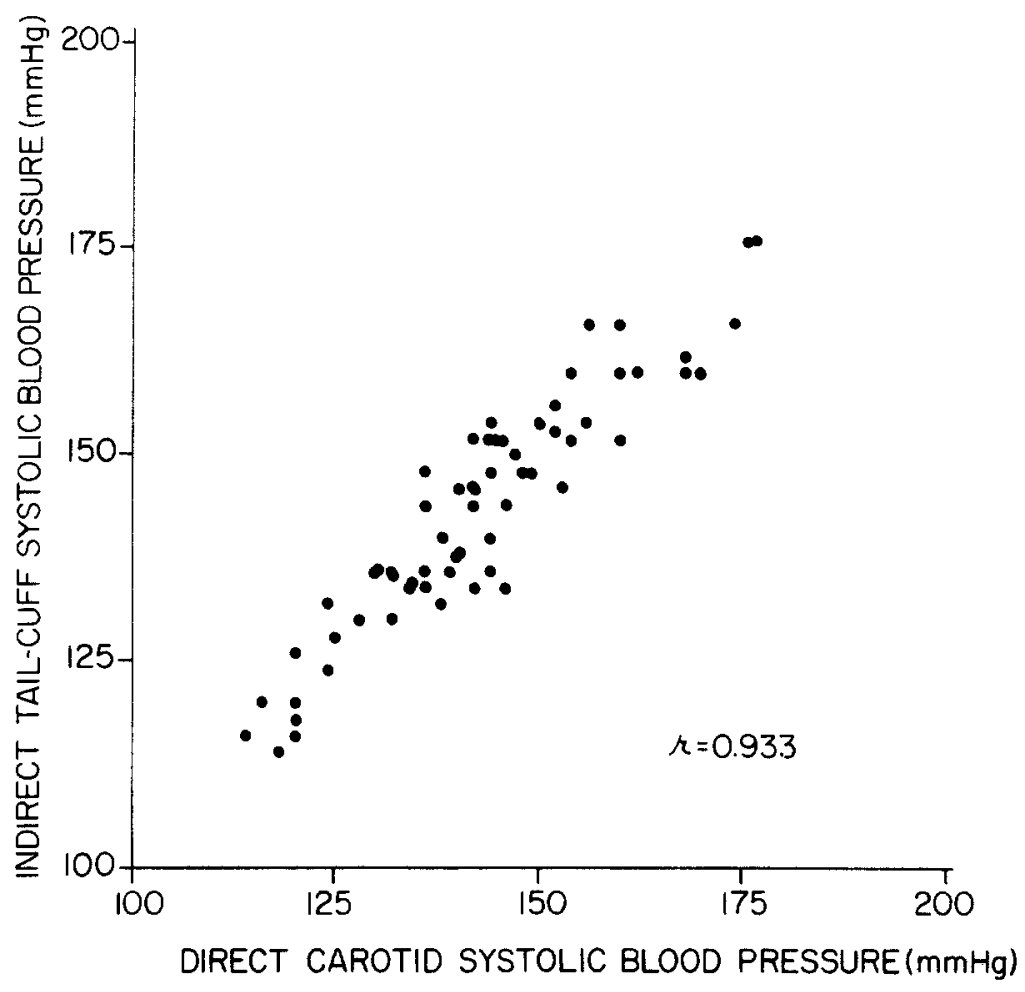

FIG. 2. Relationship of 63 simultaneously recorded direct (X-axis) and indirect ( $\mathrm{Y}$-axis) systolic blood pressure in 7 pentobarbitalanesthetized, unrestrained rats.

avoidance testing. During either of the two adaptation periods, baseline running activity of the two higher BP groups, SHR and WKYN-2, did not differ significantly from one another but was greater than that of the lowest BP group, WKYN-1. Although WKYN-2 rats were more active than WKYN-1s on both Day $1 \quad(p<0.05)$ and Day 2 $(p<0.01)$, activity differences between SHR and WKYN-1 reached significance only on Day $2(p<0.02)$. This effect results from the increased adaptation running scores for both SHR and WKYN-2 animals during the second period $(p<0.025)$, while the low scores for the WKYN-1 group remained the same between the two 30 min periods.

\section{Avoidance Acquisition}

Avoidance acquisition of the three BP groups, expressed as mean percentage avoidance responses across six 10-trial blocks, is presented in Fig. 4 and summarized in Table 2. Over the four days of testing, certain rats within the WKYN strain were clearly inferior to both SHRs and other WKYNs in acquiring the running wheel Ra. On this basis, a fairly clean delineation was made within the "normotensive" WKYN group. Those WKYNs whose first session avoidance performance was characterized by scores equal to zero, and whose scores for the remaining three sessions never reached $15 \%$, were designated subgroup WKYN-1 $(n=6)$; the remainder of the WKYN rats $(n=8)$, none of whom qualified under these criteria, were designated subgroup WKYN-2.

A Kruskal-Wallis one-way analysis of variance of the mean percent avoidance responses emitted, the frequency of pulsed shock delivery, and running scores between the three groups revealed overall significant group differences on these measures (at least $p<0.01$ for all 10-trial blocks

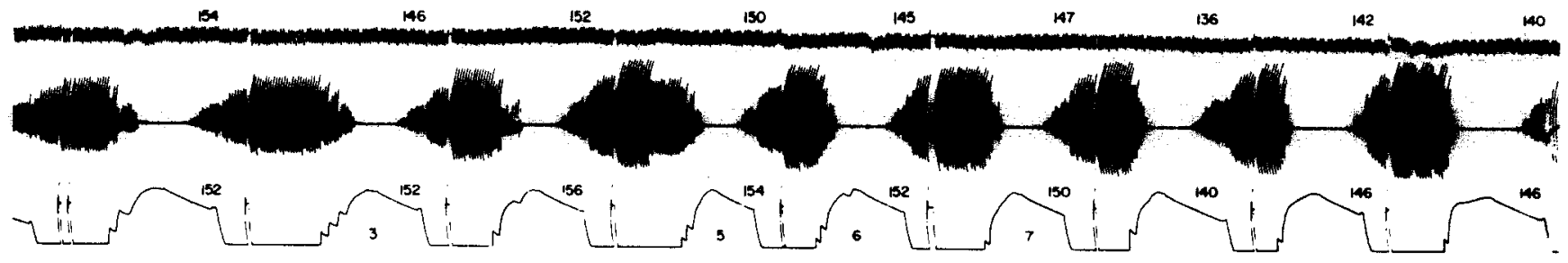

FIG. 3. Representative polygraph recording of simultaneously measured direct intra-arterial and indirect tail-cuff blood pressures in an unrestrained anesthetized rat. Upper tracing, direct carotid arterial pressure; middle tracing, caudal artery pulsations: lower tracing, intraocclusion tail-cuff pressure. Recorder stopped between cycles. 
TABLE 2

BLOOD PRESSURE, MOTOR ACTIVITY AND CAR PERFORMANCE IN NORMOTENSIVE AND SPONTANEOUSLY HYPERTENSIVE WISTAR-KYOTO RATS

\begin{tabular}{|c|c|c|c|c|c|c|c|c|}
\hline Group & $\begin{array}{l}\text { Tail Blood } \\
\text { Pressure } \\
\text { (mm Hg) }\end{array}$ & Day & $\begin{array}{l}30 \text { Min } \\
\text { Adaptation } \\
\text { Score* }\end{array}$ & $\begin{array}{l}\text { Avoidance } \\
\text { Session } \\
\text { (Day) }\end{array}$ & $\begin{array}{c}\text { \% Avoidance } \\
\text { Responses } \\
\text { Per }\end{array}$ & $\begin{array}{l}\text { Pulsec } \\
\text { Deli } \\
\text { lock o }\end{array}$ & $\begin{array}{l}\text { Shocks } \\
\text { vered } \\
\text { Ten Tr }\end{array}$ & $\begin{array}{l}\text { Running } \\
\text { Score** } \\
\text { als }\end{array}$ \\
\hline \multirow{4}{*}{$\begin{array}{l}\text { SHR } \\
(n=11)\end{array}$} & \multirow{4}{*}{$200 \pm 3.8 \dagger$} & \multirow{2}{*}{1} & \multirow{2}{*}{$702 \pm 1648$} & 1 & $63.5 \pm 2.4$ & \multicolumn{2}{|c|}{$7.3 \pm 1.5 \dagger$} & $2719 \pm 298$ \\
\hline & & & & 2 & $84.7 \pm 2.3$ & \multicolumn{2}{|c|}{$2.8 \pm 0.51 \dagger$} & $3318 \pm 310$ \\
\hline & & \multirow{2}{*}{2} & \multirow{2}{*}{$1454 \pm 316 \dagger$} & 3 & $80.8 \pm 2.5$ & \multicolumn{2}{|c|}{$4.5 \pm 0.71 \dagger$} & $4668 \pm 329$ \\
\hline & & & & 4 & $79.4 \pm 3.8$ & \multicolumn{2}{|c|}{$4.2 \pm 0.76 \dagger$} & $5084 \pm 329$ \\
\hline \multirow{4}{*}{$\begin{array}{l}\text { WKYN-2 } \\
(n=8)\end{array}$} & \multirow{4}{*}{$161 \pm 7.4 \dagger \ddagger$} & \multirow{2}{*}{1} & \multirow{2}{*}{$1120 \pm 291+\S$} & 1 & $45.6 \pm 9.5$ & 12 & $\pm 2.8 \dagger$ & $2584 \pm 435$ \\
\hline & & & & 2 & $76.3 \pm 7.2$ & \multicolumn{2}{|c|}{$4.8 \pm 1.8 \dagger$} & $2703 \pm 453$ \\
\hline & & \multirow{2}{*}{2} & \multirow{2}{*}{$2276 \pm 404 \dagger$} & 3 & $71.7 \pm 6.7$ & \multicolumn{2}{|c|}{$7.7 \pm 2.1 \dagger$} & $3865 \pm 619$ \\
\hline & & & & 4 & $74.6 \pm 6.2$ & \multicolumn{2}{|c|}{$6.0 \pm 1.5 \dagger$} & $4608 \pm 635$ \\
\hline \multirow{4}{*}{$\begin{array}{l}\text { WKYN-1 } \\
(n=6)\end{array}$} & \multirow{4}{*}{$123 \pm 4.9$} & \multirow{2}{*}{1} & \multirow{2}{*}{$377 \pm 56$} & 1 & $0.0 \pm 0.0$ & 28 & \pm 3.5 & $728 \pm 36$ \\
\hline & & & & 2 & $2.8 \pm 1.1$ & 27 & \pm 4.0 & $758 \pm 64$ \\
\hline & & \multirow{2}{*}{2} & \multirow{2}{*}{$343 \pm 58$} & 3 & $3.1 \pm 2.2$ & 58 & \pm 18 & $1177 \pm 83$ \\
\hline & & & & 4 & $1.7 \pm 1.1$ & 82 & \pm 28 & $1145-52$ \\
\hline
\end{tabular}

All values expressed as mean \pm standard error.

*A running activity score of 88 is equivalent to 1 full wheel revolution.

†Significantly differs from WKYN-1.

†Significantly differs from SHR.

\$Significant difference, Day 1 v. Day 2.

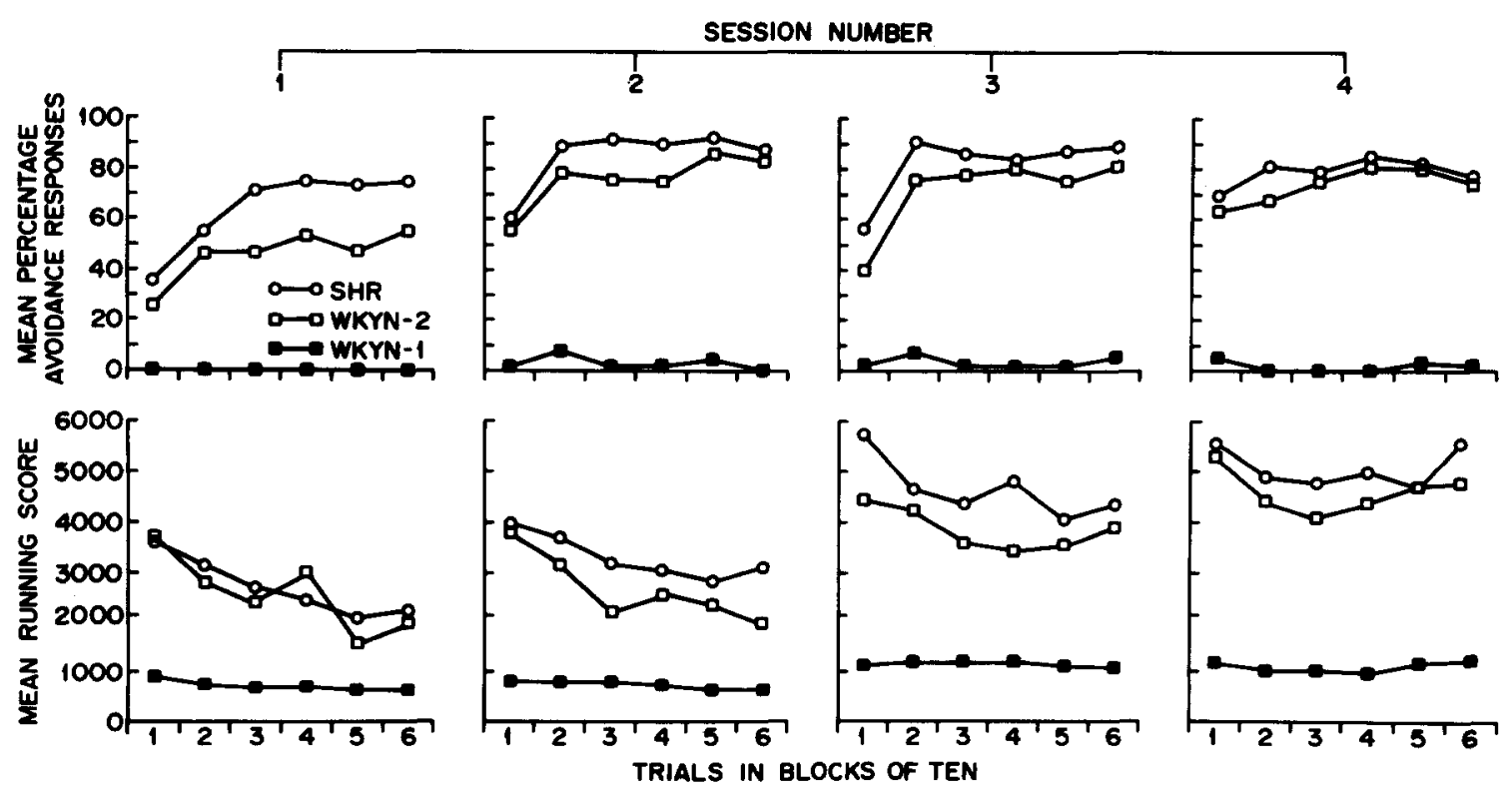

FIG. 4. Avoidance acquisition and wheel-running activity of the three blood pressure groups (WKYN-1, WKYN-2 and SHR), expressed as mean percentage avoidance responses and mean running activity score across six 10-trial blocks. 
through all four sessions). In separate Mann-Whitney U comparisons, highly significant differences in avoidance performance were observed between the lower BP - poor avoider WKYN-1 rats and the SHRs $(p<0.001)$ and between the WKYN-1 and the higher BP - good avoider WKYN-2 subgroup $(p<0.001)$ on each of the four test sessions. When tested over blocks of 20 trials, mean percent avoidance responding was reliably greater in SHRs than in the WKYN-2 rats on Trials 21-40 $(p<0.01)$ and $41-60$ $(p<0.03)$ on Day 1 , and on trials $1-20(p<0.03)$ on Day 3 (Fig. 4), but was not significantly different for these two groups across other trials in these sessions, nor during Sessions 2 or 4 . Since the avoidance criterion is first introduced to the animals on Day 1, and new criterion set on Day 3, it appears that the SHRs meet these criteria, i.e., acquire the avoidance task, more rapidly than the WKYN-2 subgroup, as well as reaching a consistently higher level of avoidance performance through the four days of acquisition testing (Table 2, Fig. 4). Although the number of pulsed shocks taken by SHRs, as indicated in Table 2, was consistently less than that received by WKYN-2 rats, this did not differ significantly on any one of the four days of acquisition testing. In contrast, the WKYN-1 subgroup took more pulsed shocks than either SHRs $(p<0.002)$ or WKYN-2 rats $(p<0.01)$ during each of the four avoidance sessions. Nevertheless, since 260 pulsed shocks would be delivered per block of ten trials if neither an $\mathrm{Ra}$ nor an $\mathrm{Re}$ were made, and since the WKYN-1 rats took only $12-32 \%$ of this number of shocks (Table 2), it appears that this subgroup did exhibit an intact Re.

Consistent with findings for avoidance performance and pulsed shock delivery, the running activity scores for both of the higher BP groups, SHR and WKYN-2, were significantly higher $(p<0.005$ for both) than scores attained by the lowest BP WKYN-1 group (Fig. 4) across all four avoidance sessions. In addition, running activity for the SHR group was, for the most part, greater than that for the WKYN-2 group, although significant differences $(p<0.05)$ were reached between these two groups only when analyzed in 20-trial blocks, in Trials $21-60$ on Day 2 and in Trials 1-40 on Day 3 (Fig. 4). The relatively low running activity of the WKYN-1 subgroup during acquisition is consistent with the low rates exhibited previously by this group during adaptation. As Fig. 4 and Table 2 demonstrate, the mean running scores for WKYN-1 rats were just above those required for successful escape responding, i.e., just enough to terminate shock: On Days 1 and 2, when the minimum running criterion was 440 ( $=5$ wheel revolutions) per block of ten trials, WKYN-1s scored 728 and 758 , respectively, while on Days 3 and 4 , when the criterion was doubled to 880 , their wheel-running activity scores were 1177 and 1145 , respectively.

\section{DISCUSSION}

In a study of the Maudsley strains of Reactive (MR) and Non-Reactive (MNR) rats, both Broadhurst and Levine [3], and Gupta and Holland [15] indicated that the acquisition of the conditioned shuttle-box avoidance responses was greater in MNR rats than in MRs, and that inferior avoidance performance of the MR strain might be due to their freezing and crouching behavior which interferes with active avoidance acquisition. The fact that MNR rats, which are more active than MR rats, show better active avoidance conditioning than $M R$ rats, suggested that the more active
SHRs would also show better active avoidance conditionme than the less active WKYNs.

The present results clearly demonstrate that those kat within the Wistar-Kyoto foundation stock exhibiting higher systolic BPs also exhibit greater motor activity and superior levels of discriminated avoidance learning across four consecutive daily sessions in a running-wheel. SHR:s acquired the CAR rapidly during the first session, taking very few pulsed shocks. Those WKYNs with high BPS tended to have an avoidance learning profile similar to that of SHRs, while WKYNs with BPs approximately $50-100 \mathrm{~mm} \mathrm{Hg}$ lower than the SHR range failed to acquire the avoidance task, yet routinely exhibited an intact escape response. Neither the design of this experiment nor the statistical analyses were of the kind needed to conclusively demonstrate heritability. Nevertheless the trend of percentage avoidance performance scores in successive generation(s) of selection (i.e., SHR, compared to the ancestral WKYNs, both WKYN-1 and WKYN-2 subgroups), suggests that there exists a heritable component in the CAR acquisition rate and performance level coinciding with, and possibly related to, the previously demonstrated $[16,36]$ heritable component of $\mathbf{B P}$ in animals of the Wistar/Kyoto stock.

This suggested type of avoidance learning inheritance is similar to that reported by Bignami [1] for a randomly bred line of Wistar-derived rats selected for high and low rates of avoidance conditioning. It is interesting, in light of the present results, that the similarity in these two Wistar-derived strains of rats extends to their BPs also: the RHA/N strain, selectively bred for high active avoidance performance, has significantly higher BPs at $4,6,8$ and 10 weeks of age than the RLA/N strain, selectively bred for a low level of avoidance learning. This evidence suggests that the genetic factors involved in this particular behavioral pattern are also related to elevated $\mathrm{BP}[16]$. The low avoidance, low BP WKYN-1 subgroup in the present investigation had lower running activity scores than the higher avoidance, higher BP WKYN-2 and SHR groups during the two-day adaptation period and during CAR acquisition. Likewise, the low avoidance animals in the Bignami study appeared to be less active than the animals of the high avoidance strain |11. In a later study, Holland and Gupta confirmed that these high avoiders (RHA/N) were more active, as measured by ambulation scores, than the low avoidance strain (RLA/N) in both open field and activity cage test situations [17]. Additional support for the generality of the present results is strengthened by a recent study of Sidman non-discriminated avoidance in SHRs [35]. After more than 15 test training sessions in the standard Sidman paradigm, normotensive WKYN rats were classified as good-, and poor- or nonperformers. Good avoiders included $40 \%$ of the total trained WKYNs, while the remaining $60 \%$ consisted of poor-and nonperformers. In contrast, almost all SHRs were good-avoiders, taking fewer shocks/min and emitting more Ra's than WKYNs.

Although Eichelman et al. [9], have reported significant differences in shock-induced jump thresholds for SHRs and WK Y Ns $(0.14$ and $0.22 \mathrm{~mA}$, respectively) the variation in intensity at this level is very likely not sufficient to account for the observed differences in avoidance performance in the present investigation because the current intensity (1.0 $\mathrm{mA}$ ) of the electric shock US was well above threshold for both strains. Consequently, assuming that the aversion threshold was similar for the WKYN-1, WKYN-2 and SHR 
groups, another explanation must be sought for observed sub-strain differences in CAR. One plausible explanation is that the drive level associated with the stressful/painful CS/US pairing in the good avoider-high BP SHR and WKYN-2 groups might be the result of the psychophysiological makeup of these animals per se, i.e., the reported hyperactivity of their sympathetic and peptidergic-hormonal systems. More explicitly, tone/shock pairings produce (1) an increased sympathetic outflow, and (2) increased availability of hypophyseal polypeptide hormones. Behaviorally, these effects together increase visceral responsivity as well as central alerting in the SHR and WKYN-2 groups as a heightened anticipatory stress response to a threatened painful stimulus. Absent or greatly reduced in the lower BP - poor avoider WKYN-1 group, these two physiological effects would, in addition, be present as an ongoing (baseline) responsivity to environmental stimuli and would be responsible for the preexisting heightened motor activity of the higher BP animals. The WKYN-1's tendency to freeze on CS presentation reflects either an absence or suppression of behavioral activation. Thus, it may be postulated that the superior avoidance performance, high running activity and perhaps even the hypertension, of the SHR and WKYN-2 groups, as compared to the normotensive WKYN-1 group, can be attributed to this two-fold physiological differentiation between them. Evidence that the hypophyseal polypeptides, ACTH, MSH and vasopressin are associated with improved CAR acquisition [8,39] and increased $B P[6,14$, $20,25,29,30]$ as is SNS activity $[5,7,11,12,19,22,24$, $28,33,38$ ] supports this hypothesis.

Recent evidence has shown that several hypophyseal polypeptide hormones, such as $\mathrm{ACTH}, \mathrm{MSH}$, vasopressin and related peptides, facilitate acquisition of and retard extinction of conditioned avoidance responding at least partially via a direct effect on the central nervous system (CNS) $[8,39]$. One plausible mode of action of these polypeptide hormones in the CNS is described in terms of the motivational property of conditioned environmental cues, and the range of stimulus cues to which an animal attends [2,18]. Thus, it has been suggested by Bohus [2] that enhancement of the association of environmental signals with motivational states (i.e., central alerting) might account for some of the behavioral effects of these hormones.

The relatively rapid extinction of avoidance responding in surgically-sympathectomized dogs [33] suggests that internal physiological (visceral) reactions are necessary for maintenance of behavior without obvious reinforcement, and further indicates that the visceral reactions themselves may act as reinforcers. Based on her behavioral studies in immuno-sympathectomized mice, Wenzel characterized immuno-sympathectomized animals as less reactive to threatened aversive stimuli, as in avoidance conditioning [38]. She further suggested that the results obtained with the immuno-sympathectomized preparation are compatible with the hypothesis that sympathetically innervated responses play a mediational role in avoidance learning. This interpretation is supported and extended by the results of the present investigation: our findings suggest that the higher sympathetic/peptidergic-hormonal activity SHRs (as well as the higher BP ancestral WKYN-2 rats) are correspondingly more reactive to threatened aversive stimuli and therefore conditioned well, while the lower activity WKYN-1 animals are less reactive to these stimuli and, consequently, conditioned poorly.

If increased sympathetic-adrenomedullary and pituitaryadrenocortical activity is related to both high blood pressure and susceptibility to stomach lesions, then an analogy might be drawn with a study by Sines et al. [32] . These workers found that a Sprague-Dawley selectively bred substrain of stomach lesion-susceptible rats are more active in an open field and reach active avoidance learning criteria significantly faster than control Sprague-Dawleys.

As a working hypothesis related to the findings reported here, the following can be said: Increased availability of pituitary polypeptides, coinciding with increased SNS activity, is genetically determined as an anticipatory hyperreactivity in hypertensive rats of Wistar-Kyoto foundation stock. This is responsible, at least in part, for the high level of open field and running-wheel motor activity and CAR performance, and is a major contributing factor in the development of hypertension, given the permissive role of the vasculature under increased pressure loads (e.g., resulting from early chronic excesses of catecholamines and adrenocorticoids). It remains for future investigation to determine what common central region or pathway, if any, is involved in the presumed genetic defect leading to this hyperreactivity of pituitary-polypeptidergic and peripheral catecholaminergic systems, and whether there exists the presently postulated causal relationship between these and the physiological/behavioral observation of high blood pressure and superior advance learning.

\section{ACKNOWLEDGEMENTS}

This research was supported, in part, by Grant MH 21403 from the National Institute of Mental Health and Grant 73-774 from the American Heart Association. The authors gratefully acknowledge the assistance of Homer Ferguson, Ronald Derman and Julio Baldrighi and the instructive and constructive critique of the manuscript by Drs. Linda Wilson and Roger Wilson.

\section{REFERENCES}

1. Bignami, G. Selection for high rates and low rates of avoidance conditioning in the rat. Anim. Behav. 13: 221-227, 1965.

2. Bohus, B. Pituitary-adrenal influences on avoidance and approach behavior of the rat. Prog. Brain Res. 39: 407-420, 1973.

3. Broadhurst, P. L. and S. Levine. Behavioral consistency in strains of rats selectively bred for emotional elimination. $B r . J$. Psychol. 54: 121-125, 1963.

4. Buffag, R. D. Validation in awake rats of a tail-cuff method for measuring systolic pressure. J. appl. Physiol. 34: 279-282, 1973.
5. Campbell, J. C., D. S. Robinson and D. Whitehorn. Differences in central nervous system regulation of blood pressure in spontaneously hypertensive and matched control rats. The Physiologist 19: 147 (Abstract), 1976.

6. Christy, N. P. Adrenal cortical steroids in various types of hypertension. In: Hormones and Hypertension, edited by W. M. Manger. Springfield, IL: Thomas, 1966, pp. 169-187.

7. De Champlain, J. Hypertension and the sympathetic nervous system. In: Perspectives in Neuropharmacology, edited by S. H. Snyder. New York: Oxford University Press, 1972, pp. 215-265. 
8. De Wied, D. Effects of peptide hormones on behavior. In Frontiers in Neuroendocrinology, edited by W. F. Ganong and L. Martini. New York: Oxford University Press, 1969. pp. 97- 140 .

9. Eichelman, B., W. De Jong and R. B. Williams. Aggressive behavior in hypertensive and normotensive rat strains. Physiol. Behav. 10: $301-304,1973$.

10. Elias, M. F. and G. Schlager. Discrimination learning in mice genetically selected for high and low blood pressure: Initial findings and methodological implications. Physiol. Behav. 13: $261-267,1974$.

11. Engelman, K., B. Portnoy and A. Sjoerdsma. Plasma catecholamine concentrations in patients with hypertension. Circulation Res. 18: $141-146,1970$.

12. Folkow, B., M. Hallbäck, Y. Lundgren, R. Silvertsson and L. Weiss. The importance of adaptive changes in vascular design for the establishment and maintenance of of primary hypertension, as studied in man and in spontaneously hypertensive rat. In: Spontaneous Hypertension: Its Pathogenesis and Complications, edited by K. Okamoto. New York: SpringerVerlag, 1972, pp. 103-114.

13. Folkow, B., M. Hallbäck and L. Weiss. Cardiovascular responses to acute mental "stress" in spontaneously hypertensive rats. Clin. Sci. Mol. Med. 45: 131s-133s, 1973.

14. Goodman, L. S. and A. Gilman (Editors). The Pharmacological Basis of Therapeutics. New York: Macmillan, 1975.

15. Gupta, B. D. and H.C. Holland. An examination of the effects of stimulant and depressant drugs on escape/avoidance conditioning in strains of rats selectively bred for emotionality/ nonemotionality. Psychopharmacology 14: 95 --105, 1969.

16. Hansen, C. T. A genetic analysis of hypertension in the rat, In: Spontaneous Hypertension: Its Pathogenesis and Complications, edited by K. Okamoto. New York: Springer-Verlag, 1972 , pp. $13-17$.

17. Holland, H. C. and B. D. Gupta. Some correlated measures of activity and reactivity in two strains of rats selectively bred for differences in the acquisition of a conditioned avoidance response. Anim. Behav. 14: 574-580, 1966.

18. Kastin, A. J., L. W. Miller, R. Nockton, C. A. Sandman, A. V. Schally and L. O. Stratton. Behavioral aspects of melanocytestimulating hormone (MSH). Prog. Brain Res. 39: 461-469. 1973.

19. Louis, W. J., A. E. Doyle, S. N. Anavekar and K. G. Chua. Sympathetic activity and essential hypertension. Clin. Sci. Mol. Med. 45: 119s-121s, 1973 .

20. Molteni, A., P. A. Nickerson, J. Latta and A. C. Brownie. Hypertension in rats bearing an adrenocorticotrophic hormone-growth hormone and prolactin - secreting tumor (MITF4). Cancer Res. 32: 114-118, 1972.

21. Mowrer, O. H. On the dual nature of learning - A reinterpretation of "conditioning" and "problem-solving." Harvard Educational Rev. 17: 102-148, 1947.

22. Nagatsu, T., T. Kato, Y. Numata (Sudo), K. Ikuta, H. Umezawa, M. Matsuzaki and T. Takeuchi. Serum dopamine$\beta$-hydroxylase activity in developing hypertensive rats. Nature 251: $630-631,1974$.

23. Okamoto, K. Spontaneous hypertension in rats. Int. Rev. exp. Pathol. 7: 22-270, 1969.
24. Okamoto. K. (Editor). Spontaneous Hyperensient lis /ath genesis and Complications. New York: Springer-Verta, 19;

25. Oliver, J. T., M. K. Birmingham, A. Bartova, M. P. Li and T. H Chan. Hypertensive action of 18 -hydroxydeosycorticosterom: Science 182: $1249-1251,1973$

26. Pappas, B. A., D. A. V. Peters, M. Saari, S. K. Sobrian and L. Minch. Neonatal 6-hydroxydopamine sympathectomy in normotensive and spontaneously hypertensive rat. Pharm. Biochem. Behav. 2: 381 -386, 1974.

27. Pfeffer, J. M., M. A. Pfeffer and E. D. Frohlich. Validity of an indirect tail-cuff method for determining systolic arterial pressure in unanesthetized normotensive and spontaneously hypertensive rats. J. Lab. Clin. Med. 78: 957--962, 1971.

28. Roizen, M. F., V. Weise, H. Grobecker and I. J. Kopin. Plasma catecholamine and dopamine- $\beta$-hydroxylase activity in spontaneously hypertensive rats. Life Sci. 17:283-288, 1975.

29. Sakamoto, A. and K. N. Prasad. The newly observed catecholamine-like actions of $\beta$-melanocyte-stimulating hormone. In: Protein and Polypeptide Hormones, Part 2, edited by M. Margoulies. Excerpta Medica Found. Int. Cong.. Ser. No. 161, 1968, pp. $503-504$

30. Scoggins, B. A., J. P. Coghlan, E. J. Cran, D. A. Denton, S. K. Fan, J. G. McDougall, C. J. Oddie, P. M. Robinson and A. A. Shulkes. Experimental studies on the mechanism of adrenocorticotrophic hormone-induced hypertension in the sheep. Clin. Sci. Mol. Med. 45: 269s 271 s, 1973.

31. Shimamoto, K. and A. Nagaoka. Behavioral and pharmacological characteristics of the spontaneously hypertensive rat. In: Spontaneous Hypertension: Its Pathogenesis and Complications, edited by K. Okamoto. New York: Springer-Verlag, 1972 , pp. $86-88$.

32. Sines, J. O., C. Cleeland and J. Adkins. The behavior of normal and stomach lesion susceptible rats in several learning situations. J.genet. Psychol. 102: 91-94, 1963.

33. Solomon, R. L. and L. C. Wynne. Traumatic avoidance learning. Psychol. Rev. 61: 353 -..385, 1954.

34. Tabei, R., T. Maruyama, M. Kuanda and K. Okamoto. Morphological studies on endocrine organs in spontaneously hypertensive rats. In: Spontaneous Hypertension: Its Pathogenesis and Complications, edited by K. Okamoto. New York: Springer-Verlag, 1972, pp. $185-193$.

35. Takaori, S., C. Tanaka and K. Okamoto. Relationship between behavior and brain monoamines in spontaneously hypertensive rats. In: Spontaneous Hypertension: Its Pathogensis and Complications, edited by $\mathrm{K}$. Okamoto. New York: SpringerVerlag, 1972, pp. 89-92.

36. Tanase, H., Y. Suzuki, A. Oooshima, Y. Yamori and I. Okamoto. Further genetic analysis of blood pressure in the spontaneously hypertensive rat. In: Spontaneous Hyper. tension: Its Pathogenesis and Complications, edited by $\mathbf{K}$. Okamoto. New York: Springer-Verlag, 1972, pp. 9-12.

37. Weiss, J. A. A tail electrode for unrestrained rats. J. exp. Anal. Behav. 10: $85-86,1967$

38. Wenzel, B. M. Immuno-sympathectomy and behavior. In: Immunosympathectomy, edited by $\mathrm{G}$. Steiner and $\mathrm{E}$. Schönbaum. Amsterdam: Elsevier, 1972, pp. 199-219.

39. Zimmerman, E., W. H. Gispen, B. H. Marks and D. de Wied (Editors). Drug effects on neuroendocrine regulation. Prog. Brain Res. 39: $407 \quad 470,1973$. 